\title{
Advanced Glycation End Product
}

National Cancer Institute

\section{Source}

National Cancer Institute. Advanced Glycation End Product. NCI Thesaurus. Code C106312.

Nonenzymatically glycosylated proteins or lipids which accumulate in vascular tissue during aging and at an accelerated rate in diabetes. 